\title{
Histological Findings of a Local Adipofascial Flap That Was Implanted during Breast Conserving Surgery
}

\author{
Yuko Kijima*, Heiji Yoshinaka, Munetsugu Hirata, Akihiro Nakajo, Hideo Arima, Sumiya Ishigami, \\ Shinichi Ueno, Shoji Natsugoe
}

Department of Digestive Surgery, Breast and Thyroid Surgery, Kagoshima University Graduate School of Medical and Dental Sciences, Kagoshima, Japan.

Email: "'ykijima@m3.kufm.kagoshima-u.ac.jp

Received June $8^{\text {th }}, 2012$; revised July $10^{\text {th }}, 2012$; accepted August $15^{\text {th }}, 2012$

\begin{abstract}
We report a patient with breast cancer whose breast was immediately reconstructed using a local adipofascial flap and was then subsequently resected 3 years after the original surgical procedure due to local recurrence. In order to achieve local control of the solitary recurrent lesion, we resected the remnant breast, which gave us a useful opportunity to examine the previously implanted flap histologically. A 33-year-old Japanese female was diagnosed with T2N0M0 breast cancer in the outer area of her left breast and underwent partial mastectomy with immediate reconstruction using a local adipofascial flap. The breast lesion was considered to be invasive ductal carcinoma, scirrhous carcinoma, lymphatic invasion+, venous invasion-, estrogen receptor+, progesterone receptor+, and HER2/neu-. Involvement was noted in three of the twenty-five resected axillary lymph nodes. She received adjuvant hormone therapy, but developed a solitary local recurrence of the skin forty-five months after the initial procedure, for which she received total mastectomy, systemic chemotherapy, and hormone therapy. During the histological examination of the local adipofascial flap that had been implanted into the partial breast defect, normal fatty tissue and the implanted fascia were seen at the implantation site. This is a rare report in which a local flap that was implanted during oncoplastic breast surgery was histologically examined.
\end{abstract}

Keywords: Breast Cancer; Oncoplastic Surgery; Local Adipofascial Flap; Cosmesis; Thoracodorsal Adipofascial Flap

\section{Introduction}

Oncoplastic surgery has recently become more popular in the world wide. It ranges from simple operations to complex procedures involving plastic techniques [1]. An oncoplastic breast-conserving one-stage surgical procedure using the volume-replacement technique is one of the most common procedures and can be performed by breast surgeons as well as plastic surgeons. Although cosmetic evaluations of this procedure have been reported by many authors, no pathological findings have been documented because clinical samples are only available when a secondary resection is selected to achieve local control after disease recurrence. We report a rare case of a breast cancer patient who underwent volume replacement oncoplastic surgery as an initial treatment followed by a total mastectomy to achieve local control of a recurrent lesion 45 months after the first procedure, and we also report the histological findings of the implanted local adipofascial flap.

*Corresponding author.

\section{Case Report}

In June 2005, a 33-year-old Japanese female was diagnosed with a breast lesion, which was classified as T2N0M0 Stage IIA according to the TNM classification [2]. Her breasts were relatively small and without ptosis. Computed tomography (CT) and bone scintigraphy showed no distant metastases, and the levels of tumor markers CEA, CA15-3, and BCA225 were within the normal ranges. As magnetic resonance imaging (MRI) and ultrasonography (US) showed that the lesion was confined to the quadrant area, we considered that breast conserving therapy (BCT) was indicated. In our institution, we generally perform two types of oncoplastic sur gery; the first type is volume replacement oncoplastic surgery for patients with small breasts, and the second type is reduction type oncoplastic surgery for patients with large and ptotic breasts. The former type was selected for this patient due to the size and volume of her breasts. An oblique incision was made on the outer area of her left breast, and a semi-circular incision on the areolar was added to facilitate the medial surgical proc- 
ess (Figures 1(a) and (b)). A C-shaped local adipofascial flap composed of subcutaneous fatty tissue and the fascia of the anterior serratus muscle was harvested just after the partial mastectomy. Then, the sheath of the muscle was attached to the adipose tissue to make the harvested tissue firm. The flap was then rotated medially to fill the cylinder shaped deformity made by the partial mastectomy. The fixation of the flap to the edge of the remnant gland was performed using PDS sutures. Based on a histological diagnosis, the breast lesion was considered to be invasive ductal carcinoma, scirrhous carcinoma, lymphatic invasion+, venous invasion-, estrogen receptor+, progesterone receptor + , and HER2/neu-. Involvement was noted in three of the twenty-five resected axillary lymph nodes. As the pathological margin from the edge of resected gland to the nearest intraductal cancer lesion was over $10 \mathrm{~mm}$, delivering additional radiation therapy to the remnant grand was avoided. LH-RH agonist (3.6 $\mathrm{mg} / 4$ weeks) and Tamoxifen (20 mg/day) were added as a hormone therapy. At 45 postoperative months, local recurrent lesions were found on the skin close to the periareoral incision scar. Systemic examination using CT, bone scintigraphy, ultrasonography, and PET showed a solitary recurrence on the remnant breast without any other distant metastases. We performed total mastectomy on July 2009 (Figure 1(c)) and shaved the pectoral major muscle thinly just under the recurrent area. Mastectomy was performed without any trouble related to the previous volume replacement treatment, such as fatty tissue necrosis, cystic degeneration, or fibrous degeneration.

The recurrent lesions were histologically restricted to the dermal to subdermal area (Figure 2). The boundary line between the remnant gland and the implanted adipofascial flap was clearly recognized histologically (Figures 3(a) and (b)). Normal fatty tissue containing cells with a clear cytoplasm was observed in the implanted area. Also, thin fibrous tissue, which was thought to be the attached fascia of the serratus muscle, was noted on top of the muscular tissue (Figure 3(c)). No degenerative changes, cystic formation, or calcification were seen in the implanted local adipofascial flap.

\section{Discussion}

BCT has become the standard treatment for invasive breast carcinomas of up to $5 \mathrm{~cm}$ in diameter and is being increasingly used for DCIS and larger tumors. Breast conserving surgery for breast cancer has two main goals: 1) radical excision of the cancer while; 2) avoiding unpleasant cosmetic results. Unfortunately, a permanent conflict exists between these two goals. In order to remove the cancer with adequate radicality (and hence reduce the risk of local recurrence), excision of large volumes of tissue is required, increasing the risk of unpleasant cosmetic results. This conflict has been well documented in the literature [3]. Partial mastectomy in which large amounts of tissue are resected increases the risk of a poor final aesthetic result if no partial breast reconstruction is performed $[3,4]$.

Postquadrantectomy deformities include localized defects in the skin and glandular tissue, distortion and/or dislocation of the areola, and retraction of the breast tissue. Perhaps the most prominent and frequent cosmetic
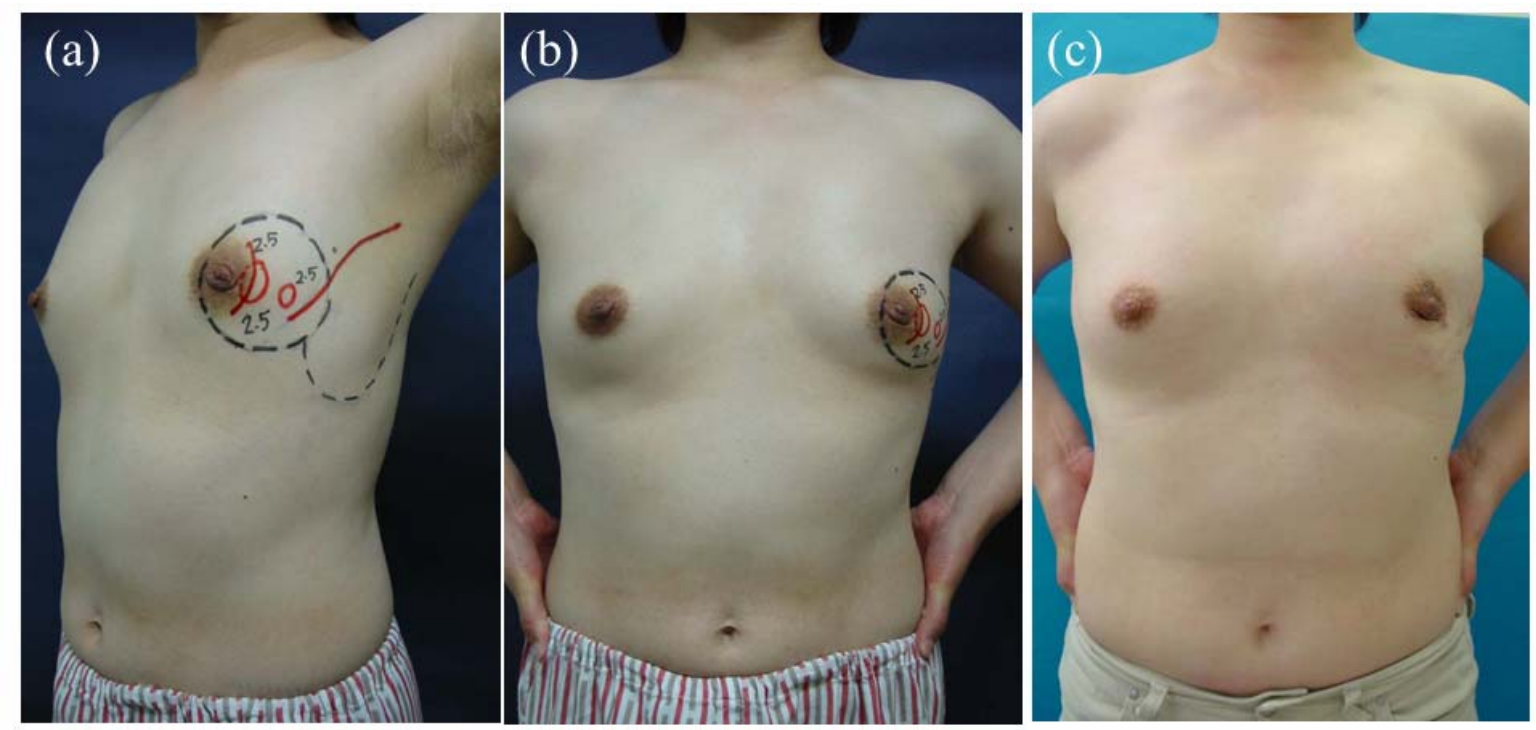

Figure 1. A 33-year-old patient. Preoperative marking of the resected area. (a), (b) The lesions were detected by ultrasonography and marked with circles. A semi-circle was drawn on the periareolar in addition to an oblique line, which served as incision lines. A C-shaped dotted line was drawn to mark the borders of the local adipofascial flap to be implanted into the medio-cranial defect. (c) Two years postoperatively. The partial defect is inconspicuous from the anterior view. 


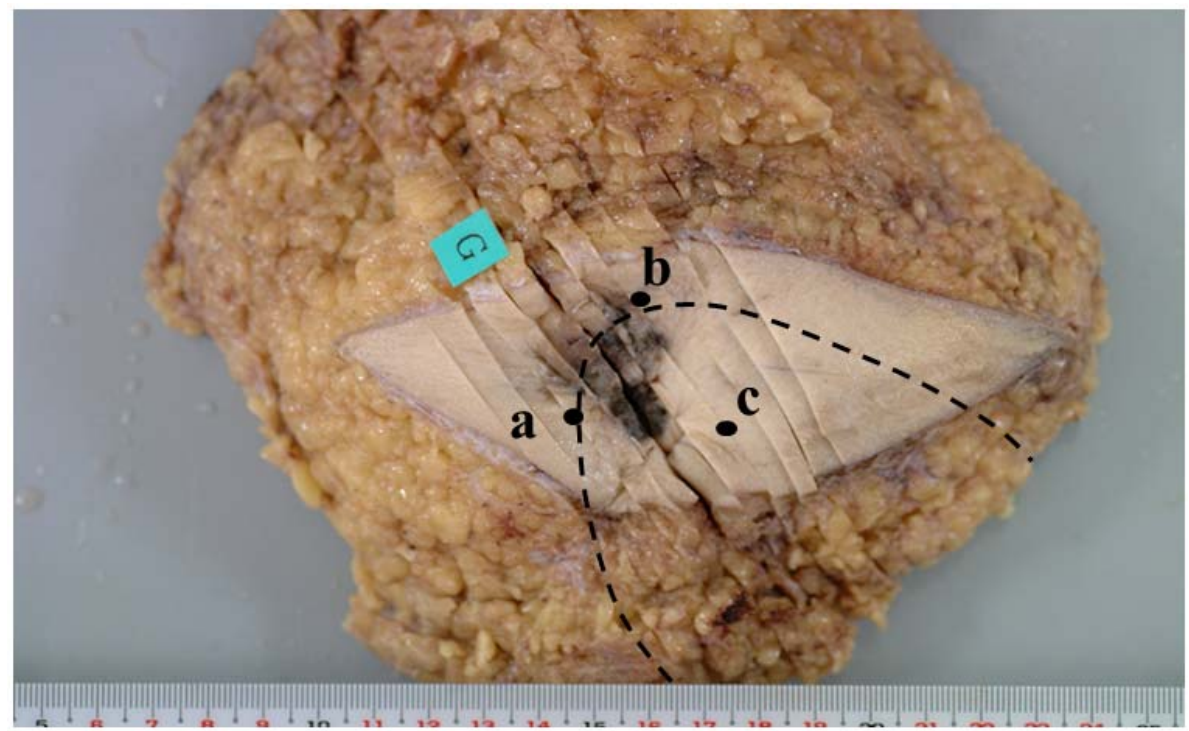

Figures 2. The resected breast at the time of local recurrence. The dotted line shows the border of the remnant gland and the local adipofascial flap implanted during the initial operation. The flap was histologically recognizable. The microscopic findings detected at points (a), (b) and (c) are shown in Figure 3.
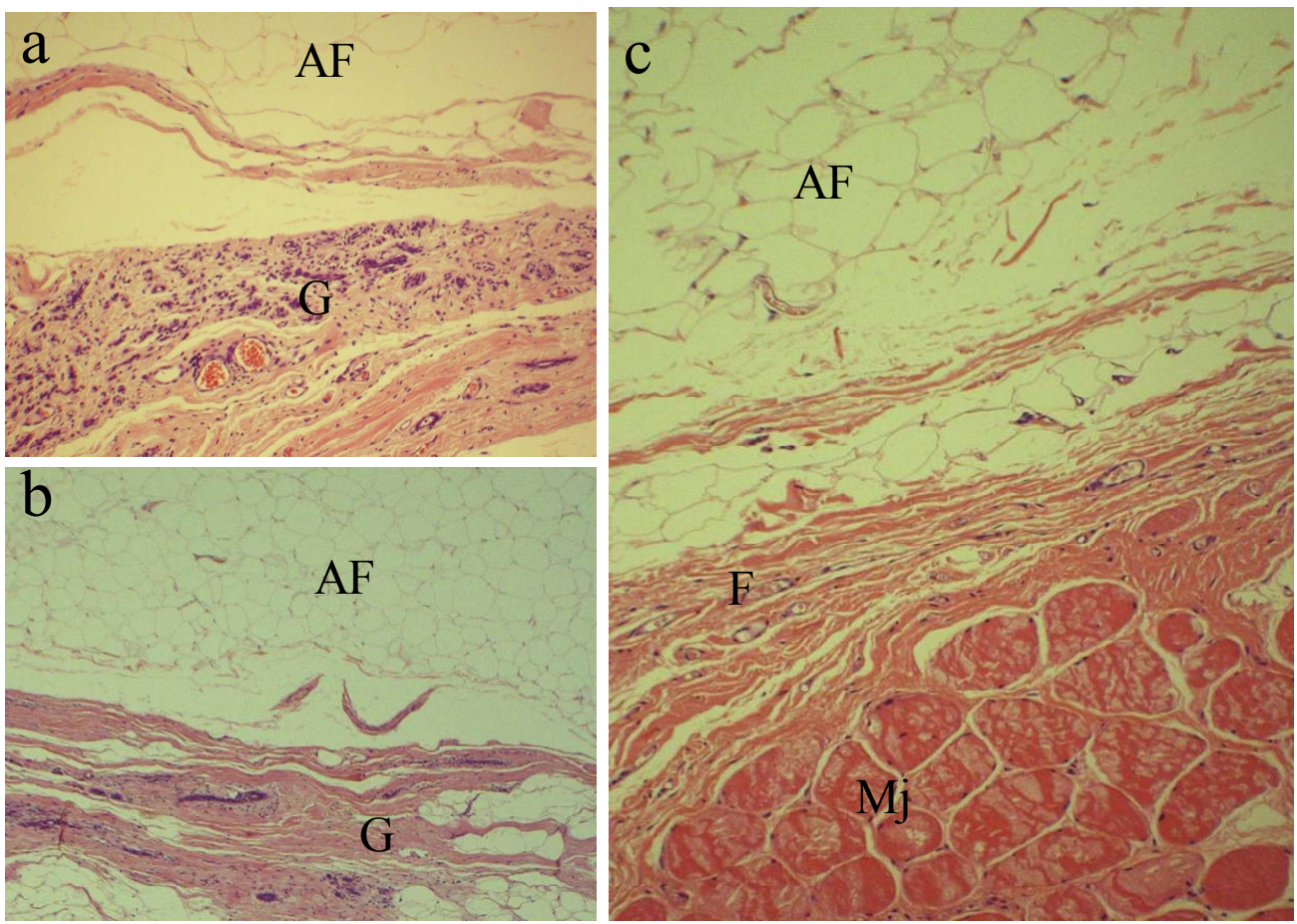

Figures 3. Marginal area of the remnant gland (G) and the implanted local adipofascial flap (AF). There was no necrotic tissue, cystic degeneration, or calcification. The implanted fatty tissue appeared normal. (HE staining, 100× magnification). (c) Deep border of the implanted area. Implanted adipofascial flap (AF), the fascia of the serratus muscle (F) and the shaved major pectoral muscle (Mj) were clearly distinguished (HE staining, 200× magnification).

failure is a lack of symmetry of the breasts. Surgical techniques used to address the conflicts between oncological and cosmetic results can be classed under the general term of oncoplastic surgery [4], which is a new surgical approach that allows wide excision but prevents breast deformities by reconstructing large resection de- fects immediately. Oncoplastic surgical procedures have been discussed in previous reports. There are two fundamentally different approaches: volume-replacement procedures that combine resectioning with immediate reconstruction of the defect using autologous tissue in the form of local fasciocutanous and volume-replacement 
procedures that combine resectioning with a variety of different breast-reduction and -reshaping techniques, according to the location of the tumor [5-7]. However, no previous clinical reports have clarified the histological findings of the implanted autologous tissue. It is easy for clinicians to observe postoperative findings of implanted tissue by physical examinations such as US, CT, or MRI, and they rarely perform additional surgical treatments, unless a recurrent lesion is restricted to the remnant gland and surgical treatment is selected.

Fortunately, we experienced a rare case of recurrence after BCT, which gave us an opportunity to histologically examine the previously implanted flap. As a result, we found that volume replacement surgery using a local adipofascial flap is safe, at least for the first 4 postoperative years. Any additional conclusive evidence that oncoplastic surgery using the volume replacement technique is safe should be reported.

\section{Results}

Oncoplastic surgery; immediate breast reconstruction using local thoracodorsal adipofascial flap, is useful for patients with small breasts because of pathological proof in this Japanese experience.

\section{Conflict of Interest Statement, Approval of Ethics Committee}

We declare that there are no financial relationships or other interests with regard to this manuscript that might be construed as constituting a conflict of interest for any author. We obtained approval for these experiments from the committee for Clinical Research at the Graduate School of Medical and Dental Sciences, Kagoshima University. Written informed consents were obtained before primary and secondary surgical treatments.

\section{Conclusion}

Oncoplastic surgery combining partial mastectomy and immediate volume replacement therapy using a local adipofascial flap was demonstrated to be safe by a clinical case in which an additional mastectomy was required.

\section{REFERENCES}

[1] J. Hoffmann and D. Wallwiener, "Classifying Breast Cancer Surgery: A Novel and Complexity-Based System for Oncological, Oncoplastic and Reconstructive Procedures, and Proof of Principle by Analysis of 1225 Operations in 1166 Patients," BMC Cancer, Vol. 9, 2009, p. 108. doi:10.1186/1471-2407-9-108

[2] L. H. Sobin and C. H. Wittwkind, "TNM Classification of Mlaignant Tumours," 6th Edition, John Wiley \& Sons, New York, 2002, pp. 31-41.

[3] B. Fisher, J. Dignam, N. Wolmark, E. Manounas, J. Costantino and W. Poller, et al., "Lumpectomy and Radiation Therapy for the Treatment of Intraductal Breast Cancer: Findings from National Surgical Adjuvant Breast and Bowel Project B-17," Journal of Clinical Oncology, Vol. 16, No. 2, 1998. pp. 441-452.

[4] P. Berrino, E. Campora and P. Santi, "Postquadrantectomy Breast Deformities: Classification and Techniques of Surgical Correction," Plastic Reconstructive Surgery, Vol. 79, No. 4, 1987, pp. 567-572. doi: 10.1097/00006534-198704000-00010

[5] C. Nos, A. Fitoussi, D. Bourgeois, A. Fourquet, R. J. Salmon and K. B. Clough, "Conservative Treatment Lower Pole Breast Cancers by Bilateral Mammoplasty and Radiotherapy," European Journal of Surgical Oncology, Vol. 24, No. 6, 1998, pp. 508-514. doi:10.1016/S0748-7983(98)93356-X

[6] Y. Kijima, H. Yoshinaka, T. Owaki, Y. Funasako and T. Aikou, "Immediate Reconstruction Using Inframammary Adipofascial Flap of the Anterior Rectus Sheath after Partial Mastectomy," American Journal of Surgery, Vol. 193, No. 6, 2007, pp. 789-791.

[7] Y. Kijima, H. Yoshinaka, Y. Funasako, K. Kaneko, M. Hirata, S. Ishigami, et al., "Immediate Reconstruction Using Thoracodorsal Adipofascial Flap after Partial Mastectomy," Breast, Vol. 18, No. 2, 2009, pp. 126-129. doi:10.1016/j.breast.2009.02.006 